\title{
El trail running (carreras de o por montaña) en España. Inicios, evolución y (actual) estado de la situación Trail runningin Spain. Origin, evolution and current situation; natural areas
}

\author{
Jordi Segui Urbaneja, Estela Inés Farias \\ Instituto Nacional de Educación Física de Catalunya (España)
}

\begin{abstract}
Resumen. Es objeto de este artículo explicar, desde un punto de vista descriptivo, los inicios y evolución del trail running (carreras de o por montaña) en España con el objetivo de determinar la existencia (o no) del boom del trail running. Se realiza el análisis cuantitativo de las carreras de trail running organizadas en el año 2015 (n=1901) a partir del cual se identifica que: a) la carrera a pie es la tipología de actividad deportiva más utilizada; b) las épocas en que más eventos se organizan son los periodos de primavera y otoño; c) Cataluña es la comunidad autónoma que más carreras organiza; d) los municipios de menos de 5000 habitantes son los que más pruebas acogen; e) atendiendo a la evolución en la creación de nuevas pruebas se observan tres periodos: 1) del 1940 hasta 1977, con un crecimiento nulo; 2) del 1978 hasta el 2006, con un ligero crecimiento y 3) del 2007 hasta el 2015, con un boom en la creación de nuevas pruebas; y finalmente f) se observa que en la creación de una prueba, mayoritariamente, se inicia con una distancia, posteriormente así como avanza en ediciones, aumenta el número de pruebas para a partir de la cuarta edición volver a una única distancia. Con estos resultados este es el primer estudio que demuestra la existencia del boom del trail running, a la vez que realiza una fotografía de la situación del trail running en España en el 2015 y de cómo ha llegado hasta aquí.
\end{abstract}

Palabras clave: boom del trail running; trail running; eventos deportivos; medio natural.

Abstract. This article explains the beginnings and evolution of trail running in Spain from a descriptive point of view. The objective is to determinate the existence of the term trail running boom. The study is based on a quantitative analysis of trail running races organized in the year 2015 ( $\mathrm{n}=1901$ ). The results are: a) foot race is the most used type of trail running; b) spring and autumn are the two periods in which more events are organized; c) Catalonia is the autonomous community that more trail running races organizes; d) municipalities of less than 5000 inhabitants organize the highest number of trail running races; e) considering the evolution in the creation of new trail running races, three periods are observed: 1) from 1940 to 1977, with no growth; 2) from 1978 to 2006, with a slow growth and 3) from 2007 to 2015, with a boom in the creation of new races; and finally f) we could observe that the organization of trail running races usually starts with only one distance; successively, in following editions the number of modalities increases, whereas from the fourth edition again it comes back to a single distance. This is the first study that demonstrates the existence of the trail running boom, as well as it explains the situation of trail running in Spain in 2015 and how it has reached its current state.

Keywords: trail running boom; trail running; sporting event.

\section{Introducción}

Las carreras de montaña, las carreras por montaña y el trail running son prácticas deportivas que en España y durante los últimos años han sido motivo de estudio y análisis atendiendo el impacto social, mediático, ambiental y económico que han y están generando en la sociedad y el territorio.

Algunos de los ámbitos que han empezado a ser estudiados por la comunidad científica, son investigaciones vinculadas a: 1) la disertación de la nomenclatura utilizada para definir la práctica o modalidad deportiva (Seguí, Inglés, Labrador, Farías; 2016); por ello y a efectos de este artículo se utilizará el termino trail running, como genérico, para referirse indistintamente a carreras de montaña (especialidad deportiva de la Real Federación Española de Atletismo-RFEA-) y/o carreras por montaña (especialidad deportiva de la Federación Española de Deportes de Montaña y Escalada-FEDME-); 2) el impacto ambiental, en este caso empiezan a ser considerables los estudios realizados por varias organizaciones, públicas -Universidades, Parques naturales, etc.- y privadas -Federaciones deportivas- (Benayas et al., 2015; Europarc España, 2016) y 3) el impacto social (Farías, E.I., Seguí, J. \& Inglés, E., 2015).

Pero, si algo tienen en común la mayoría de estudios vinculados al trail runnig es que justifican su interés atendiendo que en los últimos años se ha producido un boom del trail running, vinculado a un incremento de practicantes y de organización de eventos deportivos de dicha práctica.

La utilización del término boom del trail running fue utilizado, en el ámbito académico, por primera vez por Zagalaz, Latorre y Cachón (2002) y secundado posteriormente por Venero (2007) y, a partir de aquí, ha sido replicado, sin ser demostrado si es un hecho real o no, en el ámbito periodístico, comercial y académico. Fechada la primera utilización del término en los inicios del 2000, una década después el término sigue siendo utilizado sin haber sido científicamente contrastado. Así, durante la década transcurrida entre el inicio de la utilización del término

Fecha recepción: 15-03-17. Fecha de aceptación: 19-05-17 Jordi Segui Urbaneja

jsegui@inefc.es hasta la actualidad son varios los datos que, de manera indirecta, parecen verificar la existencia del boom del trail running: la encuesta de hábitos deportivos en España -número de practicantes no federados vinculados a la actividad física y salud-(1), el historial de licencias deportivas en España-número de practicantes federados, vinculados al deporte competición, federativo o no,- y clubes deportivos en España -número de organizaciones vinculadas al desarrollo y promoción del trail running(2) y otros estudios parciales relacionados -número de carreras, oficiales o no- (3).

\section{Encuesta de Hábitos Deportivos en España}

En 2015 se publicaba la Encuesta de Hábitos Deportivos en España 2015, Ministerio de Educación Cultura y Deporte, 2015, (CSD, 2017b) aportando datos que, de manera indirecta, pueden explicar y avalar la existencia y repercusión del boom del trail running:

a) si se atiende al porcentaje de población de 15 años en adelante que dice practicar deporte en el último año, más de la mitad (53,5\%) lo hizo. Si se observa la evolución de la población que declaró practicar deporte en el último año en las encuestas de 2005 y 2010, se observa un crecimiento de practicantes pasando del 37\% en 2005, al 43\% en 2010 hasta el 53,5\% en 2015.

b) si se refiere a la modalidad deportiva practicada en el último año, destaca la práctica del senderismo y montañismo (31,9\%) y carrera a pie (30,4\%) siendo la tercera y cuarta, respectivamente, modalidad más practicada en el último año. Si se atiende a la evolución, respecto al año 2010, se observa que la práctica del senderismo y montañismo ha sufrido un incremento del 21,4\% entre el periodo comprendido entre los años 2010 - 2015 (del 10,5\% al 31,9\% respectivamente), pasando de la octava modalidad más practicada en 2010 a la tercera en 2015. La práctica de carrera a pie también sufre un incremento del 6,6\% entre los años 2010 y 2015 (del 23\% al 30,4\% respectivamente), pasando de segunda (2010) a tercera (2015) modalidad más practicada. Mientras que en el año 2005 la encuesta de hábitos deportivos no registro este campo.

c) si se observa la modalidad deportiva más frecuente practicada semanalmente, destaca la carrera a pie $(10,6 \%)$ y el senderismo, montañismo (4,9\%) siendo la segunda y séptima, respectivamente, modalidades más practicadas. Si se atiende a la evolución, respecto al 
año 2010, se observa que la práctica de la carrera a pie ha aumentado el 5,7\% entre el periodo comprendido entre los años 2010 - 2015 (del 4,9\% al 10,6\% respectivamente), pasando de la cuarta modalidad más practicada en 2010 a la segunda en 2015. La práctica de senderismo y montañismo también sufre un incremento del 3\% entre los años 2010 2015 (del 1,9\% al 4,9\% respectivamente), pasando de doceava (2010) a séptima (2015) modalidad más practicada. Mientras que en el año 2005 la encuesta de hábitos deportivos no registro este campo.

d) si se fija la atención en el lugar donde suele practicar deporte, se observa que el $45,9 \%$ de practicantes lo hacen en el aire libre, siendo este el principal espacio de práctica. No existen datos de los años 2005 y 2010 puesto que la encuesta de hábitos deportivos no registro este campo.

e) finalmente, si se considera el porcentaje de personas que disponen al menos de una licencia deportiva en vigor en el último año, se observa que este representa el 9,8\% siendo el grupo de edad de entre 15 y 24 años el más numeroso (20\%).

Por lo tanto, se puede concluir que: 1) se ha producido un aumento de la población que dice practicar deporte en el último año; 2) la práctica del senderismo y montañismo y la carrera a pie son dos de las modalidades más practicadas en los últimos años y también de las más frecuentemente practicadas semanalmente; 3) la práctica al aire libre es el principal espacio de práctica; y 4) la mayoría de practicantes realizan actividad física sin estar vinculados (mediante licencia deportiva) a una federación deportiva. Todo ello parece indicar que, efectivamente, se ha producido un aumento de práctica en el aire libre relacionado con las modalidades de senderismo y montañismo y la carrera a pie desvinculada de la actividad federativa.

\section{Historial de licencias deportivas y clubes deportivos en España}

Cada año el Consejo Superior de Deportes (CSD, 2017a) publica en su web oficial el histórico de licencias deportivas (a) y clubes deportivos (b). Si de ambas bases de datos se analiza la evolución de las dos federaciones deportivas que organizan las competiciones oficiales a nivel estatal relacionadas con el trail running: la RFEA, carreras de montaña y la FEDME, carreras por montaña, se observa que:

a) si se atiende al historial de licencias de la RFEA se observa que desde 1991 es bastante estable manteniéndose, aproximadamente, sobre las 90000 licencias con una variación de +/- 15000 licencias. Si se observa el historial de licencias de la FEDME, des de 1991 hasta el año 2002 va aumentando muy lentamente de las 54104 licencias en 1991 hasta las 66466 licencias en 2002, para a partir del 2002 dispararse en el número de licencias hasta las 203860 registradas en el año 2015.

b) si se refiere al historial de clubes deportivos españoles, publicado por primera vez por el CSD en el año 2001, vinculados a las respectivas federaciones deportivas se observa que ambas federaciones nacionales, RFEA y FEDME, aumentan en número de clubes: 1) RFEA pasa de los 627, en 2001, a los 1351, en 2015; y 2) FEDME pasa de los 1222, en 2001, a los 2475 en 2015. Pero, aun aumentando ambas federaciones, resulta destacable que no lo hacen con la misma proporción, así entre 2001 y 2005 la diferencia entre RFEA y FEDME era de unos 620 clubes (+/-20), y es a partir del 2006 cuando FEDME se distancia hasta los 1124 clubes de diferencia en 2015; de manera que la diferencia en número de clubes entre RFEA y FEDME en 2015 casi se ha duplicado.

Por lo tanto, se puede concluir que: 1) tanto la RFEA como la FEDME han aumentado en número de licencias y clubes deportivos; 2) que el aumento no ha sido proporcional, así la FEDME sufre un incremento mucho mayor tanto en número de licencias como de clubes; 3) que aun siendo un aumento constante, se puede visualizar que es en 2002 el año de inflexión en el aumento de licencias deportivas y en 2005 el año de inflexión en el aumento de clubes deportivos; y 4) es la FEDME la que aparentemente, sin menospreciar otros factores (consciencia de prevención de riesgos, evolución de los seguros deportivos, incremento de la regulación normativa, etc.), se ha beneficiado del boom del trail running vinculándose más a las carreras por montaña (disciplina FEDME) que a las carrera de montaña (disciplina RFEA).

\section{Otros estudios parciales relacionados}

A lo largo de los últimos años han sido varios los estudios que con mayor o menor rigor científico han intentado, de manera parcial, ilustrar el boom del trail running en España. De éstos destacamos:

a) Pucurull (2014), tras analizar las 150 carreras de ruta más importantes y significativas de Cataluña en los años 2010-2013, y el número de corredores que participaron en las mismas, observó un aumento del 19\% entre los años 2010 y 2011; del 12\% entre 2011 y 2012; y del 2\% entre el año 2012 y 2013.

b) Ropits Social Running (2014), confirmó que, en Catalunya, de las 38 carreras registradas en 2008 aumentaron hasta las 663 en 2014, con un total de, aproximadamente, 111.500 participantes; lo que supuso una media de 12,7 carreras por semana con una participación media aproximada de 200 participantes por carrera.

c) Oñorbe (2014a), expuso la evolución exponencial del número de carreras de trail running organizadas en el Parque Nacional de la Sierra de Guadarrama (declarado en 2013), pasando de 1 prueba en el año 2012 a más de 40 en el año 2014.

d) finalmente, Europarc-España (2016) publicaba la Guía de buenas prácticas para el desarrollo de carrera por montaña en espacios protegidos, en la que aporta datos como: 1) en España se celebrarían, en el año 2015, cerca de 1900 carreras de trail running; 2) la media de participantes en estas carreras se acercaría a los 500 (destacamos, como ejemplo, laUltra Trail de MontBlanc, carrera de trail running emblemática que congrega anualmente unos 7000 corredores y unos 30000 espectadores); 3) se calcula que cerca de 700000 personas corren anualmente por espacios naturales singulares; 3) FEDME, controlaría el 23\% de las pruebas organizadas, mientras que el $77 \%$ restantes son organizadas por otras entidades (ayuntamientos, asociaciones, empresas, etc.)

Por lo tanto, se puede concluir que: 1) efectivamente se observa, en los últimos años, un incremento en el número de carreras de trail running y un incremento en el número de participantes en este tipo de eventos; y3) la organización de carreras de trail running a través de la vía federativa -FEDME- (de la RFEA no se disponen datos) es, aproximadamente, del 23\% del total de eventos organizados en España.

De los tres análisis expuestos anteriormente se puede entender que:

1) el incremento de población que dice practicar senderismo y montañismo y carrera a pie en el aire libre coincide en el tiempo con el aumento del número de licencias y clubes deportivos que, en mayor o menor medida, han sufrido la RFEA y la FEDME.

2) la mayoría de la población que dice practicar senderismo y montañismo y carrera a pie en el aire libre parece hacerlo al margen de la actividad federativa (sin licencia deportiva oficial).

3) parece haber una relación temporal entre el incremento de población practicante de senderismo y montañismo y carrera a pie en el aire libre y el aumento de las carreras de trail running aunque no se pueda afirmar categóricamente cuál de los dos actúa como hecho causante y cuál como consecuencia.

4) el incremento de licencias y clubes deportivos de FEDME sobre la RFEA parecen tener relación con que FEDME sea la vía federativa que más carreras de trail running organice, siendo la FEDME la federación deportiva española la que mejor ha recogido y asumido el boom del trail running.

5) que el $77 \%$ de carreras sean organizadas por otras entidades no federativas da margen a especular que: a) existe margen para el beneficio económico, interés empresarial; b) hay margen para el beneficio social fomentar la práctica deportiva-, interés de la administración pública; y c) puede existir un uso de las carreras de trail running como desarrollo del turismo y territorio como apuntaban las tesis de Ewert, A; Attarian, A; Hollenhorst, S; Russell, K; Voight, A. (2006).

Tras el análisis expuesto, la principal cuestión es que aun habiendo estudios parciales que intentan explicar y determinar el boom del trail running no ha habido publicación alguna que exponga datos concretos; y este, precisamente, es el objeto de este artículo, explicar el boom del trail running relacionándolo con el número y evolución de las carreras de dicha disciplina deportiva. Así se considera que las carreras se organizan 
como respuesta a la demanda de los practicantes, de manera que guardan una relación proporcional: a más practicantes, más carreras se organizarán.

Así las cosas, ¿existe realmente el boom del trail running? Son objetivos de esta investigación: 1) determinar la(no) existencia del boom del trail running; 2) conocer la evolución temporal en la creación de nuevas carreras de trail running; y 3) conocer las características de las carreras de trail running.

\section{Metodología de estudio}

La metodología utilizada en este estudio ha sido el análisis cuantitativo (ej. Neuendorf, 2002) a partir de datos secundarios obtenidos de la sistematización de bases de datos de las carreras y marchas de trail running en España registradas durante el año 2015.

Para la obtención del listado de carreras y marchas de trail running registradas durante el año 2015 se solicitó la base de datos de: el calendario oficial de carreras de la RFEA, la FEDME y de Runedia, web especializada y más popular en el sector de calendarización de eventos deportivos entre ellos trail running, la cual dispone de la base de datos más completa y extensa de carreras de España. Las dos primeras, RFEA y FEDME, no respondieron mientras que Runedia (runedia.com) respondió satisfactoriamente, facilitando el calendario de eventos del año 2015. Es a partir de la base de datos, aportada por Runedia, en la que se ha basado el estudio que se presenta a continuación.

La investigación ha consistido en la sistematización y análisis de las carreras y marchas de trail running en España a partir de los datos disponibles en la base de datos inicial configurada por los siguientes campos: nombre del evento, mes en que se realizaba, población, provincia, distancia de la carrera, número de edición, año de la primera edición y email de contacto del organizador. A partir de esta se siguieron los siguientes pasos:

- Paso 1. Se añaden los campos: comunidad autónoma (se obtiene a partir del campo inicial de provincia); número de habitantes (a partir del campo inicial de población cruzado con la base de datos del Instituto Nacional deEstadística (INE), actualizado en el año 2015); tipología de evento (se obtiene a partir del nombre de evento o verificando en la web oficial del evento) y número de distancias (se obtiene a partir del campo inicial distancia)

- Paso 2. Se filtran los eventos deportivos (campo de tipología de evento) considerando las carreras, marchas y rutas por montaña, así se excluyen las carreras de asfalto, pruebas en bicicleta, a nado, triatlones, duatlones y rutas GR.

- Paso 3. Se excluyen aquellos eventos que no transitan exclusivamente en el territorio español. Se encuentran eventos procedentes de Argentina, México, Portugal, Andorra, Francia y Estados Unidos.

La base de datos inicial contenía 2563 eventos deportivos, que tras el paso 3, finalmente se obtienen 1901 eventos, los cuales constituyen la muestra de estudio. Identificada la muestra $(n=1901)$ se traslada al programa SPSS, versión 21, para su posterior análisis estadístico:

- Paso 4. Se crean nuevos campos: agruhab (agrupa las poblaciones por número de habitantes siguiendo el criterio de población aplicado por el Instituto de Estadística de Catalunya, 2015) y aguedi (agrupa las carreras según el número de edición).

Para el análisis de la base de datos se ha realizado:

- Análisis 1. Tablas de frecuencias: por Comunidad Autónoma (número de eventos por CCAA); por mes (número de eventos por mes); tipología (número de eventos según su tipología); agruhab (número de eventos según tamaño de población) y agruedi (número de eventos según edición)

- Análisis 2. Tablas de contingencias: tipología y agruedi (para identificar la antigüedad por tipología de evento); CCAA y agruedi (para conocer la antigüedad por CCAA); CCAA y agruhab (para saber el tamaño de población por CCAA); CCAA y tipología (para relacionar el número de cada tipología hay en cada CCAA) y n ${ }^{\circ}$ distancia y agruedi (para reconocer la relación entre antigüedad y número de distancias de cada evento)

\section{Resultados}

\section{Carreras de trail running según su tipología}

De las cuatro tipologías de eventos deportivos considerados, se observa que las carreras a pie son mayoritarias $(76,4 \%)$ representando casi las tres cuartas partes del total de eventos. Cabe destacar que dentro de esta categoría quedan englobadas diferentes disciplinas como: los trails, las carreras en línea, las carreras ultra, las maratones, medias maratones, el cross y los kilómetros verticales. También destaca que el 20,7\% (393 eventos) sean marchas y caminatas (Tabla 1).

\begin{tabular}{lcc}
$\begin{array}{l}\text { Tabla } 1 . \\
\text { Carreras de trail running según la tipología }\end{array}$ & \\
\hline Tipología & Frecuencia & Porcentaje \\
\hline Carrera a pie & 1452 & 76,4 \\
Marcha & 195 & 10,3 \\
Caminada & 198 & 10,4 \\
Travesías y rutas & 56 & 2,9 \\
Total & 1901 & 100,0 \\
\hline
\end{tabular}

\section{Carreras de trail running según el mes de realización}

En relación a la distribución temporal de las carreras de trail running se observa que la mayor parte de las pruebas se concentran en los meses de primavera, marzo, abril, mayo y junio, (45,3\%) y en otoño, septiembre, octubre y noviembre, (31,6\%). Figura 1.

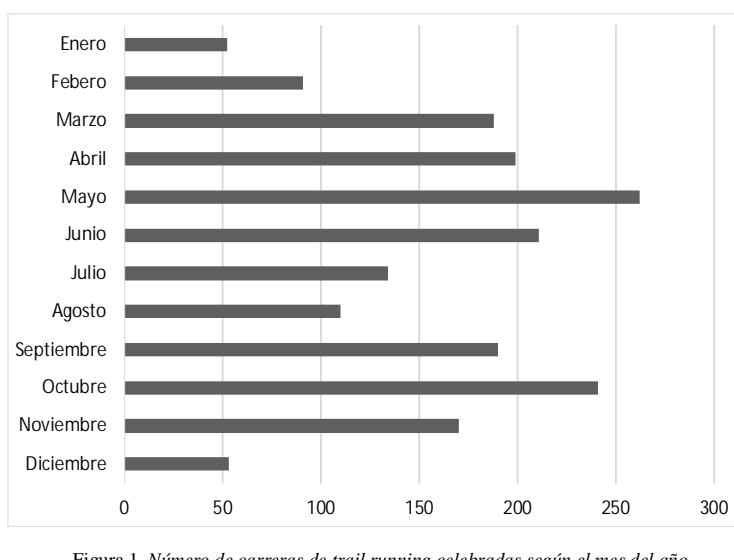

Carreras de trail running por Comunidad Autónoma

Catalunya es la comunidad donde mayor número de pruebas se organizan con un total de 819 (43,08\%). Le sigue, muy de lejos, la Comunidad Valenciana, con 180 pruebas (9,46\%) y Andalucía, con 136 pruebas (7,15\%). Posteriormente aparece un grupo de comunidades, que más o menos rondan sobre las 90 pruebas: Aragón (99 pruebas), Galicia (94), Islas Canarias (93) y Castilla y León (91). Finalmente el resto de comunidades están por debajo de las 60 pruebas anuales (Figura 2).

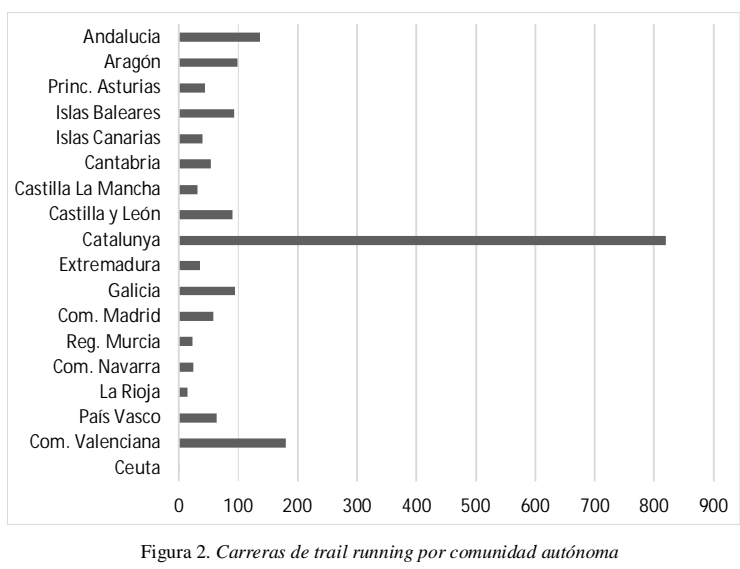


Carreras de trail running según número de habitantes del municipio

Cabe tener en cuenta que existen 55 carreras de trail running que no han podido ser vinculadas a un municipio y por lo tanto son consideradas casos perdidos; por lo tanto la muestra sobre la que se basa este análisis es de 1846 casos. A partir de aquí, se observa que, las poblaciones de menos de 5000 habitantes son las que acogen 988 pruebas (53,8\%). Por grupos de población destaca: las poblaciones de entre 1001 y 3000 habitantes, con 331 pruebas (17,4\%); las de menores de 500 habitantes, con 311 pruebas (16,4\%). A continuación aparecen las poblaciones de 10001 - 30000 habitantes, con 289 pruebas (15,2\%) y las de 5001 - 10000 habitantes, con 270 (14,2\%). Finalmente, el resto de grupo de poblaciones organizan del 10\% al 8\% de carreras (Figura3).

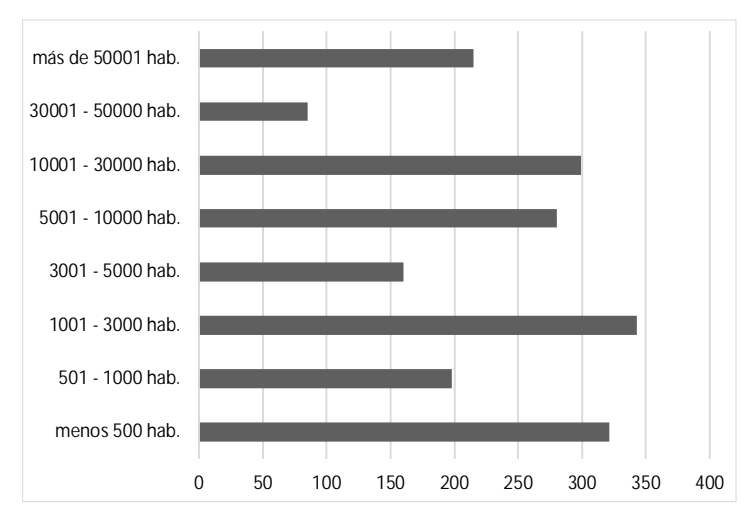

Figura 3. Carreras de trail running por tamaño del municipio

\section{Carreras de trail running según el número de edición}

Cabe tener en cuenta que existen 338 pruebas de las que se desconoce el número de edición; por lo tanto la muestra sobre la que se basa este análisis es de 1563 casos. A partir de aquí, se observa que, aun sabiendo que las carreras a pie tienen mayor número de pruebas (1228), las carreras a pie han tenido un fuerte aumento en los años 2012 - 2014 ( $4^{\mathrm{a}}-2^{\mathrm{a}}$ edición) acumulando el $41,53 \%$ de pruebas en este periodo. En relación a las marchas y las caminatas, se observa, un crecimiento, más o menos, estable en el tiempo (unas 9 nuevas carreras anuales para las marchas y unas 6 nuevas carreras anuales para las caminatas). Finalmente en relación a las travesías y rutas mantienen se observa una tendencia a la disminución en la creación de nuevas carreras (Tabla 2).

\begin{tabular}{|c|c|c|c|c|}
\hline \multirow{2}{*}{$\begin{array}{l}\text { Número de edición } \\
\text { (número y \%) }\end{array}$} & \multicolumn{4}{|c|}{ Tipología } \\
\hline & Carrera a pie & Marcha & Caminada & $\begin{array}{c}\text { Travesías y } \\
\text { rutas }\end{array}$ \\
\hline 1ª edición & $70(5,7 \%)$ & $9(5,0 \%)$ & $7(6,2 \%)$ & $1(2,3 \%)$ \\
\hline $2^{\mathrm{a}}$ edición & $159(12,9 \%)$ & $13(7,3 \%)$ & $6(5,3 \%)$ & $0(0 \%)$ \\
\hline $3^{\mathrm{a}}$ edición & $198(16,1 \%)$ & $13(7,3 \%)$ & $9(7,9 \%)$ & $6(13,9 \%)$ \\
\hline $4^{\mathrm{a}}$ edición & $162(13,2 \%)$ & $12(6,7 \%)$ & $6(5,3 \%)$ & $7(16,3 \%)$ \\
\hline $5^{a}$ edición & $124(10,1 \%)$ & $9(5,1 \%)$ & $5(4,4 \%)$ & $4(9,3 \%)$ \\
\hline $6^{\mathrm{a}}-10^{\mathrm{a}}$ edición & $277(22,6 \%)$ & $37(20,7 \%)$ & $33(29,2 \%)$ & $6(13,9 \%)$ \\
\hline $11^{\mathrm{a}}-15^{\mathrm{a}}$ edición & $97(7,9 \%)$ & $33(18,4 \%)$ & $15(13,3 \%)$ & $4(9,3 \%)$ \\
\hline Más de 15 ediciones & $141(11,5 \%)$ & $53(29,6 \%)$ & $32(28,3 \%)$ & $15(34,9 \%)$ \\
\hline Total $\left(\mathrm{n}^{0}\right)$ & $1228(100 \%)$ & $179(100 \%)$ & $113(100 \%)$ & $43(100 \%)$ \\
\hline
\end{tabular}

Según la base de datos analizada y considerando el número de edición, se identifica la marcha «Fortunata Mendi Ibilaldia» organizada en la población de Zubieta, de 323 habitantes censados, provincia de Guipúzcoa, como la prueba más antigua. Organizándose por primera vez en el año 1940, con 75 ediciones es la prueba más antigua que actualmente sigue dando el disparo de salida. Se caracteriza por ofrecer una única distancia de 46 quilómetros.

A partir de la edición del evento se puede conocer la evolución en número de carreras que se han iniciado cada año. Así se observan tres periodos: a) del 1940 hasta 1977, con un total de 13 (0,83\%) nuevas carreras; b) del 1978 hasta el 2006, con un total de 416(26,61\%) nuevas carreras y c) del 2007 hasta el 2015, donde se dispara el número de eventos con un total de 1135 (72,61\%) nuevas carreras. Si se centra la atención en los últimos nueve años (2007 - 2015) donde se crean el 72,61\% de carreras, se observan, a su vez, dos tendencias: a) una al alza durante los años 2007 al 2013 con la creación de 870 nuevas carreras y b) una a la baja, a partir del año 2014 al 2015 con la creación de 265 nuevas carreras, lo que supone una disminución en el número de nuevos eventos en los dos últimos años (Figura 4).

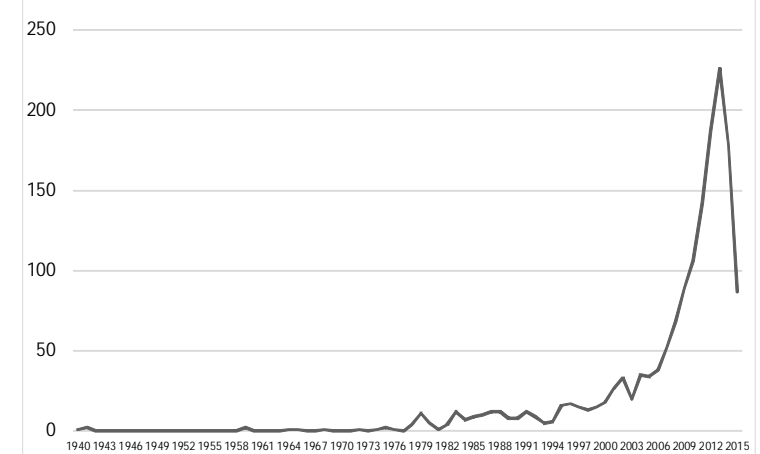

Figura 4. Evolución de nuevas carreras organizadas en el periodo 1940 - 2015

Relación entre la edición de la carrera y el número de distancias

Se observa que de las carreras analizadas ( $\mathrm{n}=1563)$ el 78,06\% (1220 pruebas) lo hacen en una única distancia; el 16,76\% (262 pruebas) ofertan dos recorridos; el 4,09\% (64 pruebas) organizan tres distancias y el $1 \%$ restante lo hace con cuatro o cinco distancias.

Si se realiza el análisis de las distancias que se ofertan en relación al número de edición de las carreras de trail running, se observa que las carreras que se organizan por primera vez tienden a hacerlo en una única distancia (62 carreras -71,26\%-). Las carreas que se organizan por segunda y tercera vez tienden a ampliar el número de distancias hasta 2 (41 y 50 eventos respectivamente) y 3 (12 y 10 eventos respectivamente). A partir de la cuarta edición se observa una disminución en el número de distancias que contemplan (Tabla 3).

\begin{tabular}{|c|c|c|c|c|c|}
\hline \multicolumn{6}{|l|}{ Número de edición } \\
\hline (número y \%) & 1 & 2 & 3 & 4 & 5 \\
\hline $1^{\text {a edición }}$ & $62(5,1 \%)$ & $18(6,9 \%)$ & $5(7,8 \%)$ & $2(15,4 \%)$ & $0(0 \%)$ \\
\hline $2^{\mathrm{a}}$ edición & $123(10,8 \%)$ & $41(15,6 \%)$ & $12(18,8 \%)$ & $2(15,4 \%)$ & $0(0 \%)$ \\
\hline $3^{a}$ edición & 165 (13,5\%) & $50(19,1 \%)$ & $10(15,6 \%)$ & $1(7,7 \%)$ & $0(0 \%)$ \\
\hline $4^{a}$ edición & $136(13,5 \%)$ & $43(16,4 \%)$ & $7(10,9 \%)$ & $1(7,7 \%)$ & $0(0 \%)$ \\
\hline $5^{\text {a }}$ edición & $108(8,9 \%)$ & $26(9,9 \%)$ & $5(7,8 \%)$ & $0(0 \%)$ & $2(50 \%)$ \\
\hline $6^{\mathrm{a}}-10^{\mathrm{a}}$ edición & $288(23,6 \%)$ & $44(16,8 \%)$ & $16(25 \%)$ & $5(38,5 \%)$ & $0(0 \%)$ \\
\hline $11^{\mathrm{a}}-15^{\mathrm{a}}$ edición & $135(11,1 \%)$ & $9(3,4 \%)$ & $4(6,3 \%)$ & $1(7,7 \%)$ & $0(0 \%)$ \\
\hline Más de 15 ediciones & $203(16,6 \%)$ & $31(11,8 \%)$ & $5(7,8 \%)$ & $1(7,7 \%)$ & $2(50 \%)$ \\
\hline Total $\left(\mathrm{n}^{\circ}\right)$ & $1220(100 \%)$ & $262(100 \%)$ & $64(100 \%)$ & $13(100 \%)$ & $4(100 \%)$ \\
\hline
\end{tabular}

\section{Discusión y conclusiones}

Este trabajo describe la evolución y las características de las carreras de trail running en España durante el año 2015 relacionándolo con el boom del trail running, lo que la convierte en un estudio pionero y una contribución novedosa, y hasta necesaria, en el ámbito de la práctica deportiva en el medio natural. A través del análisis de 1901 carreras de trail running, los resultados concretan la evolución en el número de carreras y determinan el boom del trail running además de la caracterización de las carreras en relación a la tipología de la disciplina deportiva, el mes de realización, la Comunidad Autónoma, el número de habitantes del municipio y el número de distancias que ofertan.

En primer lugar, en relación a las carreras de trail running según su tipología destaca que la mayoría sean carreras a pie, actividad más vinculada a la competición y al deporte federado; mientras que la minoría sean marchas, caminadas y travesías y rutas, actividades más vinculadas a la actividad física y la salud (al respecto cabe tener en cuenta que pueden existir muchas más marchas y travesías que no sean organizadas y por lo tanto recogidas en la fuente de datos analizada). Estos datos contrastan con el número de licencias deportivas (de la RFEA y la FEDME) y los ofrecidos por la encuesta de hábitos deportivos en 
España donde la población mayor de 15 años dice practicar deporte de las cuales la minoría (el 9,8\%) dispone al menos de una licencia deportiva en vigor en el año 2014. Tomando estos últimos datos (teniendo en cuenta todas las limitaciones en la fuente de los mismos) parece que la relación entre las carreras a pie y las marchas, caminadas y travesías y rutas debería ser inversa, así el número de carreras a pie debería ser menor respecto al número de marchas, caminadas y travesías y rutas (mayor)

En segundo lugar, en relación a las carreras de trail running según el mes de realización resulta evidente que los periodos de primavera y otoño sean los que más carreras concentran. Parte de la explicación puede venir dada atendiendo al clima Mediterráneo del territorio y al calendario laboral. La problemática puede darse considerando el impacto que las carreras de trail running pueden generar en el medio, atendiendo que estos periodos pueden coincidir con episodios de máxima floración y crecimiento, a nivel de flora, y en periodos de cría o nidificación, a nivel de fauna, (Oñorbe, 2014b).

En tercer lugar, en relación a las carreras de trail running por comunidad autónoma, sorprende la gran diferencia de la primera CCAA organizadora respecto al resto. La Guía de buenas prácticas para el desarrollo de carreras por montaña en espacios naturales protegidos (Europarc España, 2016) ya intentaron explicar este hecho vinculado a: el número de habitantes de cada CCAA, el porcentaje de territorio montañoso o el hábito de la práctica deportiva por CCAA, entre otras. Otros indicadores que pueden ser interesantes sería conocer el número de participantes por prueba, ya que aportaría datos más reales del impacto de práctica.

En cuarto lugar, en relación a las carreras de trail running según el número de habitantes del municipio, no sorprende saber que la mayoría de las carreras se organicen en poblaciones de menos de 5000 habitantes. Las hipótesis que pueden explicar este hecho son, entre otras, dos: a) los municipios cercanos a las montañas son de menos de 5000 habitantes; y b) las carreras de trail running están siendo utilizadas como producto deturismo para dinamizar el territorio rural, en la línea argumental de Martos, P. (2002). Tres son los datos que pueden justificar esta última hipótesis: 1) el impacto económico que genera una carrera de estas características. Por ejemplo, un estudio de la Consejería Insular de Economía y Hacienda de la Isla de La Palma cuantificó el impacto económico de la carrera «Ultramaratón de montaña Transvulcania» en la edición de 2013, en 3,8 millones de euros para la Isla; 2) la relación del territorio como receptor de turismo. Así, según los datos de FRONTUR (2014), las CCAA con mayor índice turístico son (de mayor a menor): Catalunya, Islas Canarias, Islas Baleares, Andalucía y la Comunidad Valenciana; territorios que coincide con las CCAA que más carreras organizan; y 3) que el 77\% de carreras sean organizadas por otras entidades no federativas da margen a especular que: hay margen de beneficio económico, empresas, hay margen de beneficio social, ayuntamientos, hay margen para desarrollar el turismo y el territorio, etc.

En quinto lugar, en relación a las carreras de trail running según el número de edición, se confirma la existencia del boom del trail running. Así se observa que en los últimos nueve años (del 2007 al 2015) se produce un aumento de casi tres terceras partes de las carreras existentes en el año 2015; dato que coincide con el incremento de práctica de hábitos deportivos de la última década y con las fechas en el incremento de licencias deportivas y el número de clubes deportivos de la RFEA, en menor medida, y FEDME, en mayor medida. Cabe destacar, también, que a partir del 2013 se observa una reducción en la creación de carreras, hecho que lleva a preguntarse si: ¿se estará estancando la creación de nuevas carreras?, ¿¿se habrá llegado a la maduración del mercado -pinchar la burbuja-? Por otro lado, parece confirmarse el estancamiento en el crecimiento de las marchas y las caminatas.

En sexto lugar, las carreras de trail running en relación al número de distancias, se observa una tendencia a que en las primeras ediciones la mayoría solamente ofertan una única distancia, en la segunda y tercera edición amplían el número de distancias que organizan, para posteriormente en las sucesivas ediciones ir disminuyendo el número de distancias, mayoritariamente, hasta finalizar con una o dos.
Finalmente y en la línea de varios autores, entre ellos Oñorbe, $\mathrm{M}$. (2014b) y Fernández Arroyo, R. (2015), cabe destacar que resulta casi imposible disponer de una cifra exacta de las carreras de trail running que se organizan en España. Según la Guía de buenas prácticas para el desarrollo de carreras por montaña en espacios naturales protegidos (Europarc España, 2016), contabilizaban 1100 pruebas que pasan por espacios naturales protegidos (basándose en el número de permisos solicitados a las administraciones ambientales), aun reconociendo que existe un porcentaje no registrado. En este estudio la muestra analizada ha sido de 1901 carreras para el mismo año (no limitadas a trascurrir por espacios naturales protegidos) y también reconocemos la limitación de considerar dicha cifra como real asumiendo que existe un número desconocido de carreras no censadas. Así, se debería mejorar la sistematización en la recogida de datos (no únicamente el número de carreras sino también el tipo de organizador, limitación de participantes, etc.) por parte de las administraciones implicadas, ambientales o municipales, y también de las respectivas federaciones deportivas. Solamente conociendo la situación (número y características) de las carreras de trail running, se podrá identificar y calcular el impacto (social, económico y medioambiental) de la actividad y tomar, si es preciso, las medidas, represivas (hard law), aconsejables (soft law) y/o correctoras en pro de una práctica deportiva que sea sostenible y sustentable temporalmente y territorialmente en todos los ámbitos.

\section{Limitaciones y perspectivas de futuro}

Son limitaciones de este estudio:

- la base de datos en la que se ha basado el estudio no incluye a todas las carreras de trail running organizadas en España durante el año 2015.

- mucho de los datos utilizados para intentar explicar los inicios, evolución y actual situación del trail running en España, proceden de fuentes diversas y han sido obtenidos con metodologías diferentes, por lo tanto, a veces resulta complicado y hasta científicamente criticable extrapolar relaciones directas.

Son perspectivas de futuro:

- cruzar las bases de datos de competiciones oficiales (RFEA y FEDME) junto a las no oficiales.

- ampliar el estudio en base a campos como: número de participantes, tipo de recorrido (circular o lineal), si transcurren por zonas naturales protegidas, naturaleza jurídica del organizador, características del personal laboral y voluntariado, el sociotipo del participante, etc.

Datos que permitirán explicar la existencia (o no) de las carreras de trail running con el impacto medioambiental, con el desarrollo del turismo rural, con el impacto económico del deporte y con el impacto sociológico

\section{Referencias}

Benayas, J., Oñobre, M., \& Horcajo, L. (2015). Propuesta de medidas de gestión ambiental para la sostenibilidad de las carreras por montaña. Universidad Autónoma de Madrid, UAM. Madrid.

Consejo Superior de Deportes, CSD (2017a). Histórico de licencias y clubes deportivos de España. Tomado el 17 de enero de 2017 en: http://www.csd.gob.es/csd/asociaciones/1fedagclub/03Lic/

Consejo Superior de Deportes, CSD (2017b). Encuesta de hábitos deportivos. Tomado el 18 de enero de 2017 en: http:// www.csd.gob.es/csd/sociedad/encuesta-de-habitos-deportivos/

Farías, E.I., Segui, J. \& Inglés, E. (2015). Profiling participants of sporting events in thenatural environment: trail running races. 1st International Conference «Sport Tourism» Sustainable Development of Sport Tourism. Portoroz.Eslovenia.

Europarc-España (2016). Guía de buenas prácticas para el desarrollo de carrera por montaña en espacios protegidos. Madrid. EuroparcEspaña. PP 102. h t t p : / / w w w . f e d m e . e s / index.php?mmod=static Content\&IDf $=450$

Ewert,A., Attarian, A., Hollenhorst, S., Russell, K. \& Voight,A. (2006). Evolving adventure pursuits on public lands: emerging challenges 
form management and public policy. Journal of park and recreation administration, 24 (2): 125-140.

FEDME Consejo Asesor Científico de las Montaña. Área de accesos y naturaleza. (2011). Incidencia Socioeconómica y Ambiental Carreras por Montaña. Medio natural y rural en España. Tomado el 24 de enero de 2017 en:http://www.magrama.gob.es/es/desarrollo-ru$\mathrm{ral} /$ temas/caminosnaturales/incidencia\%2 Bcarreras_tcm7170626.pdf

Fernández Arroyo, R. (2015). Cuando correr en montaña deja de ser un deporte amable. Quercus 347:78-81

FRONTUR (2014). Encuesta de movimientos turísticos en fronteras (Frontur). Tomado el 20 de enero de 2017 en: http:// estadisticas.tourspain.es/en-EN/estadisticas/frontur/Paginas/ default.aspx

Martos, P. (2002). El Medio Natural como Punto de Encuentro de Turismo y Deporte. Crecimiento y Diversificación. In S. Rebollo M; Simoes, E (Eds.), Nuevas Tendencias de Práctica Físico-Deportiva en el Medio Natural (pp. 3-14). Granada: Universidad de Granada.

Neuendorf, K. A. (2002). The content analysis guidebook. Sage Publications. Thousand Oaks, London.

Oñorbe, M. (2014a). Diagnóstico de la situación actual de las carreras por montaña en el Parque Nacional de Guadarrama. Tomado el 17 de enero de 2017 en: http:// manuelmedioambiente.wordpress.com/2014/07/11/ carreras_pnguadarrama

Oñorbe, M. (2014b). Los retos en la gestión de las actividades deportivas en Espacios Naturales Protegidos. Congreso Nacional del Medio Ambiente. COMANA 2014. Tomado el 20 de enero de 2017 en: http://www.conama.org/conama/download/files/conama2014/ CT\%202014/1896711249.pdf

Pucurull, M. (2014). Sigue creciendo el número de corredores. Tomado el 18 de enero de 2017 en: http://running.es/reportajes/siguecreciendo-el-número-de-corredores

Ropits Social Running S.L. (2014). Runedia. Tomado el 19 de enero de 2017 en: http://www.runedia.com

Seguí. J., Inglés, E., Labrador, V. \& Farías, E. (2016). Carreras (de o por) montaña o trail running. El reconocimiento de la modalidad deportiva. RETOS. Nuevas tendencias en Educación Física, Deporte y Recreación 30: 162-167.https://dialnet.unirioja.es/descarga/articulo/5390990.pdf

Venero, J. P. (2007). La clase de Educación Física como motor de cambio social. Reflexionando sobre actividades en la naturaleza, currículum oculto y valores. RETOS. Nuevas tendencias en Educación Física, Deporte y Recreación, 11: 51-53. https:// dialnet.unirioja.es/descarga/articulo/2257862.pdf

Zagalaz, M. L., Latorre, P. \& Cachón, J. (2002). Intervención del profesorado de educación física en el turismo activo. Actividades turístico-recreativo-deportivas en Jaén. RETOS. Nuevas tendencias en Educación Física, Deporte y Recreación, 3:17-24.

\section{Agradecimientos}

Los autores agradecen a Ropis Social Running S.L. que haya cedido, de manera desinteresada, su base de datos para el análisis que ha permitido la realización del presente estudio y con ello que haya colaborado con la sociedad científica y civil en comprender el boom del trail running en España.

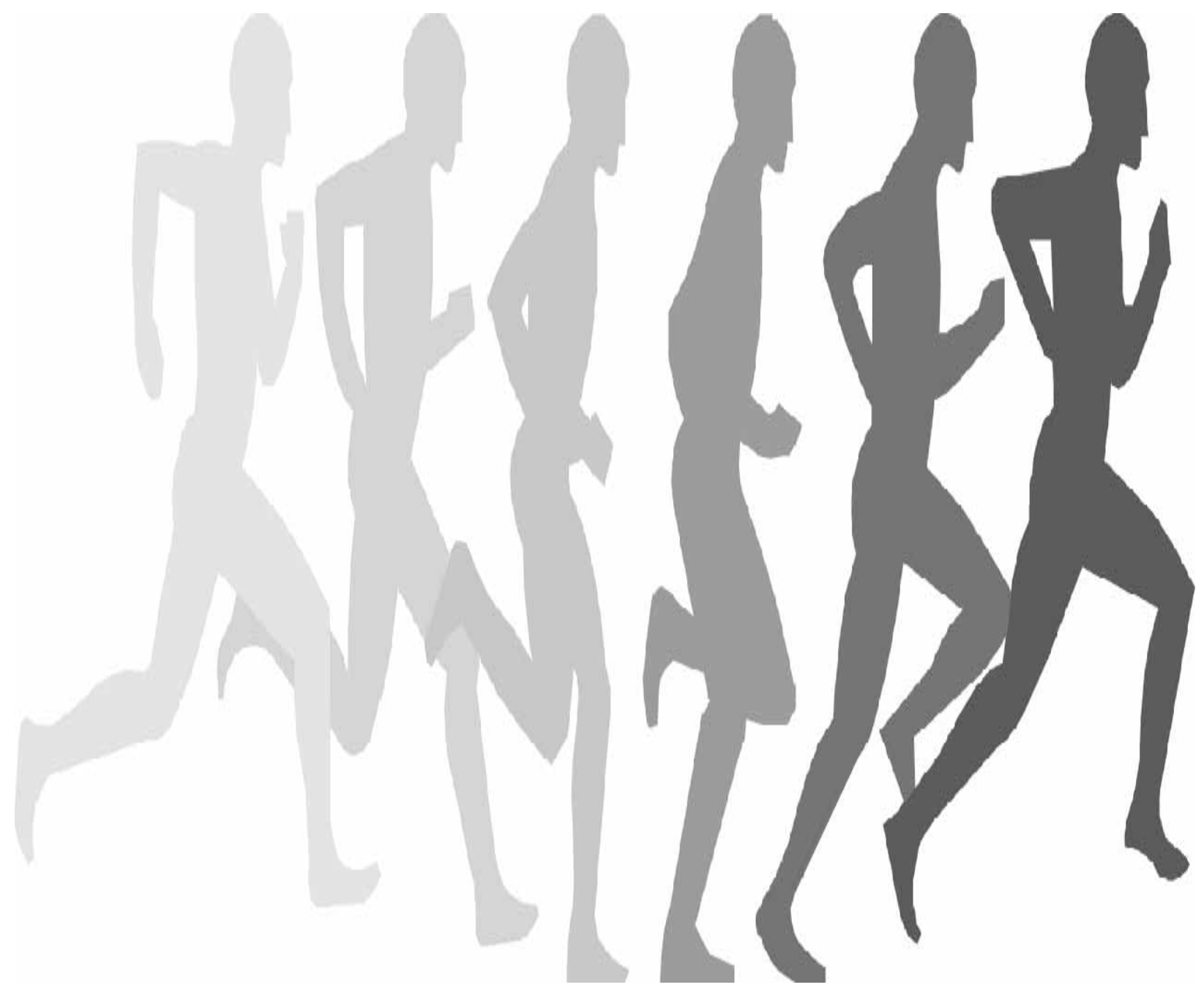

